\title{
Botulinum toxin: could it be an effective treatment for chronic tension-type headache?
}

\author{
Sherif M. Hamdy $\cdot$ Hatem Samir $\cdot$ M. El-Sayed • \\ Nermin Adel · Rasha Hasan
}

Received: 5 October 2008/ Accepted: 22 October 2008/Published online: 22 November 2008

(C) Springer-Verlag 2008

\begin{abstract}
Several clinical trials suggest that botulinum toxin type-A (BTX-A) may be an effective treatment option for patients with chronic tension-type headache (CTTH); however, controversy remains as to how the botulinum toxin optimally should be used for treating headache and which patient's profile fits this treatment. The objective of this study was to evaluate the efficacy and tolerability of BTX-A for the prophylactic treatment of CCTH in Egyptian patients. This was a randomized, singleblind, placebo-controlled study of BTX-A for the treatment of patients aged 25-50 years old with CCTH. Following a 30-day screening, headache parameters and severity assessed by the standard visual analogue scale (VAS), and the 25-item Henry Ford Hospital Headache Disability Inventory (HDI) were recorded as a baseline. Then, injection was done with either BTX-A or with saline by a combination of two methods for detecting injection sites (the fixed-site approach and follow-the-pain approach). Our study showed significant improvement after 1 month of BTX-A injection regarding headache days/month, severity measured by VAS and HDI in headache severity. There was significant reduction of prophylactic medications, and there were minor complications, but these reversed spontaneously without further treatment. BTX-A was an effective and well-tolerated prophylactic treatment in Egyptian patients with CCTH.
\end{abstract}

Keywords Botulinum toxin .

Chronic tension-type headache - Prophylactic treatment

S. M. Hamdy · H. Samir $(\bowtie) \cdot$ M. El-Sayed · N. Adel ·

R. Hasan

Department of Neurology, Cairo University, Cairo, Egypt

e-mail: samirhatem@hotmail.com

\section{Introduction}

Tension-type headache (TTH) is a distressing condition which, when chronic, can affect patients almost daily and can often be difficult to treat effectively [1]. It is the most common type of recurrent primary headache disorders that induces significant functional limitations, and imposes large individual and socio-economic burdens. Its life time prevalence in general population ranges from 30 to $78 \%$ [2]. TTH is included in the International Headache Society (IHS) classification in 2004 [3], and is further categorized as either episodic or chronic; it is also defined by whether or not associated with pericranial tenderness.

The pathophysiology of TTH remains controversial [46], pain etiology was presumed to be the muscular contraction of pain-sensitive structures of the cranium, but no research supports muscular contraction as the sole pain etiology [1]. Hence, the IHS intentionally abandoned the terms "muscular contraction headache" and "tension headache". Several other mechanisms have been proposed, including central medullary mechanisms, that involve altered stimulus response function of second-order transmission neurons in the spinal cord or in the brain stem; and central cortical mechanisms that involved disturbance of limbic pathways to the brain stem and caused by dysregulated endogenous pain-control system [4, 7].

The therapeutic effect of botulinum toxin (BTX-A) in headaches was serendipitously noted in 1992 by William Binder who identified that patients with migraine headaches recovered from their attacks following BTX-A injections for the treatment of facial wrinkles [8]. Thereafter, BTX-A was increasingly considered a promising preventive treatment for patients with refractory primary headache disorders especially migraine and chronic tension type headaches (CTTH) [7, 9-11]. However, by evaluating 
the use of BTX-A in different types of headaches in an evidence-based manner, though most of the initial reports on botulinum toxin in TTH and in migraine were positive yet, these results were contradictory in well-designed, randomized controlled trials $[12,13]$.

The proposed mechanism of effect of BTX-A in CTTH was reduction of pericranial muscle tension by cholinergic chemodenervation [14]; however, beyond this peripheral mechanism that does not fit the pathophysiological concept of all forms of headache $[11,15]$ BTX-A has an impact on the neuronal signaling pathways activated during headache, and it also exerts a blocking action on the parasympathetic nervous system, and might inhibit release of other neurotransmitters or affect transmission of afferent neuronal impulses [1]. Durham et al. [16] recognized that BTX-A could inhibit calcium-dependant release of CGRP (calcitonin gene-related peptide) from stimulated trigeminal neurons in an experimental model. Other studies have shown that BTX-A interferes with other neurotransmitters and pain modulators such as substance $\mathrm{P}$ that mediated by cleavage of the intracellular effector SNAP-25 (synaptosome-associated protein), bradykinin, and glutamate $[17,18]$.

The aim of this study was to evaluate the efficacy and tolerability of BTX-A for prophylactic treatment of CTTH in Egyptian patients.

\section{Materials and methods}

Study protocol and treatment

This was a randomized, single-blind, placebo-controlled study that was conducted on 28 Egyptian patients to determine the therapeutic effects of BTX-A BOTOX $^{\circledR}$, Allergen) in CTTH. The study protocol was designed according to the recommendations of the International Headache Society (IHS), and it was conducted with the approval of the Ethical Committee of National Cancer Institute Cairo University. A written, informed consent was obtained from all patients prior to the study commencement following an explanation of the nature, duration, and purpose of the study.

Screening phase

Prior to the study, the included patients were subjected to thorough physical and neurological examination, all of them had their routine hematological and biochemical profile that included (complete blood count, blood sugar, liver and kidney function tests), and CT scan brain. Patients were requested to keep a headache diary for 1 month prior to treatment and for 3 months posttreatment. Subjects recorded in his/her diary the following headache parameters in their provided diaries: headache duration, number of headache days per month, the start and stop times of headache, headache associated symptoms, number of days with acute headache medications were taken, and headache severity assessed by the standard visual analogue scale (VAS) from 0 (no pain) to 10 (unbearable pain) [19]. After completing the 1-month period, they were subjected to the baseline 25-item Henry Ford Hospital Headache Disability Inventory (HDI, maximum score 100) [20]. All these efficacy variables were assessed at baseline, 30 and 90 days after injection.

\section{Participant characteristics}

All participants included in this study had CTTH. The diagnosis of CTTH was established according to the criteria of the IHS (bilateral location, non-pulsating quality, with no more than one of photophobia, phonophobia or mild nausea). Eligibility criteria: patients were eligible in this study when they had (1) headache on equal or more than 15 days per month on average for at least 3 months; (2) headache duration of 1-10 years; (3) history of failed treatment in the last 3 months with at least one prophylactic drug (4) ability to distinguish between the different headache types.

\section{Exclusion criteria}

(1) Patients with migraine or other forms of primary or secondary headaches;

(2) Planned or actual pregnancy, lactation, or women of childbearing age using inadequate contraceptive measures;

(3) Any type of substance use disorders, those with drug-induced headache, and patients with medications overuse (simple analgesics, narcotic analgesics, and benzodiazepines) in the last 2 years;

(4) Previous exposure to BTX-A, any neuromuscular disease, or treatment with drugs affecting the neuromuscular junction;

(5) Prior injection of anesthetic or steroid into the muscles to be injected in the month prior to study entry;

(6) Serious physical and psychiatric disease.

When the baseline diary confirmed the inclusion criteria, the patients were randomized to receive injections of either BTXA (100 U BOTOX in $4 \mathrm{~mL}$ saline) or placebo ( $2 \mathrm{~mL} 0.9 \%$ $\mathrm{NaCl}$ vial). Patients were allocated to one of the two treatment groups by a computer-generated randomization list.

Injection technique

Patients were injected with BTX-A for only one session and followed up for 3 months. We adopted a combination 
of two methods for detecting injection sites; fixed-site approach and follow-the-pain approach [21, 22]. This treatment paradigm was used to detect the most tender points in a template of mostly affected muscles in patients with CTTH. After placing the patient in a sitting position, patients were questioned on the anatomic location of the headache. Injected tender points were identified from the potential tender points in the following muscles: frontalis, temporalis, sternocleidomastoideus, trapezius, splenius capitis, and semispinalis [22, 23]. Tender points were identified by history-taking, and by manual palpation. Intramuscular injections using sterile technique were administered using 1-mL insulin syringes. BTX-A 100-U vial was reconstituted with preservative-free $0.9 \%$ saline to yield $2.5 \mathrm{U} / 0.1 \mathrm{~mL}$. Tender points were then injected with either BTX-A or saline. The chosen dose of BTX-A varied among patients, but was the same for all injected tender points in any one patient; the dose of BTX-A for different muscles was adopted from Evers et al. [23] (Table 1), and the dose ranged from 2 to $12 \mathrm{U}$ per site. Dosage schedules were decided according to the affected muscle size taking into consideration that the doses injected in the cervical shoulder girdle muscles are kept low, so as to prevent any possible weakness [22]. Patients in the control group received equivalent volumes of saline.

\section{Outcome measures}

The primary efficacy variables were headache days per month and headache severity as measured by VAS, and quality of life assessed by HDI. Secondary efficacy variables included, duration of headache per day (hours), and number of days of symptomatic therapy per month. For all patients, reassessment was done 30 and 90 days after injection.

\section{Safety measures}

Any adverse event that a subject reported during the study was recorded by the investigators, graded for severity (mild, moderate, or severe), and assessed for its relationship to study treatment (none, possible, probable, or

Table 1 Doses and injection sites of Botulinum toxin

\begin{tabular}{ll}
\hline Dose $(\mathrm{U})$ & Muscle name \\
\hline $2 \times 2$ & M. frontalis ${ }^{\mathrm{a}}$ \\
4 & M. temporalis \\
10 & M. sternocleidomastoideus \\
12 & M. trapezius \\
10 & M. splenius capitis \\
10 & M. semispinalis \\
\hline
\end{tabular}

a Two injections sites per side definite). A serious adverse event was defined as one that was fatal, life-threatening, permanently disabling, or required admission to hospital.

Data analysis

Management of data was carried out with the Statistical Package for Social Sciences (version 12, SPSS Inc. Chicago, IL, USA). Nominal data were analyzed using either simple $\chi^{2}$ test or Fisher exact probability test, depending on sample size and expected frequencies. Ordinal measurements were analyzed using independent $t$ test. General linear model by repeated measure ANOVA was generated to assess changes of efficacy variables from baseline at 30 and 90 days. The level of significance was set at 0.05 .

\section{Results}

Population characteristics

\section{Subject demographics}

In total, 28 Egyptian patients with CTTH [9 (32.1\%) males and $19(67.9 \%)$ females] were enrolled in this study during the period from August 2006 to April 2007; and their follow-up was completed by July 2007. Participants age ranged from 25 to 50 years with a mean age of $(36.57 \pm 7.61)$ years. Out of included patients $10(35.7 \%)$ were smokers, and $3 / 19(15.79 \%)$ received contraceptive pills. Eight $(28.57 \%)$ of our patients received secondary education, 15 (53.57\%) has university graduation, and 5 $(17.86 \%)$ had a higher educational level. Patients were randomized to receive either BTX-A $(n=14)$ or placebo "saline" $(n=14)$. The demographic variables showed no differences between both groups (Table 2).

\section{Headache history}

The headache duration ranged from 1 to 10 years with a mean of $(4.79 \pm 2.57)$ years. All patients received prophylactic treatment during the last 3 months before enrollment (this includes multiple preventive strategies as antiepileptic drugs, muscle relaxants, propranolol, amitriptyline, and SSRIs), the duration of prophylactic treatment ranged from 5 to 30 months with a mean of $(16.14 \pm 7.49)$ months. Baseline clinical profile regarding headache duration, the duration of preventive therapy number of headache days per month (frequency), duration of headache per day (hours), number of days with acute headache medications were taken, headache severity measured by VAS and quality of life measured by HDI, and showed no differences between both groups (Table 3 ). 
Table 2 Baseline demographic characteristics of the study population

\begin{tabular}{llll}
\hline Variables & BTX-A & Placebo & $P$ value \\
\hline $\begin{array}{lll}\text { Age (years) } \\
\text { Sex }\end{array}$ & $36.29 \pm 7.75$ & $36.86 \pm 7.75$ & 0.847 \\
M & $4(28.6 \%)$ & $5(35.7 \%)$ & 1.0 \\
F & $10(71.4 \%)$ & $9(64.3 \%)$ & \\
Smoking & & & \\
Yes & $5(35.7 \%)$ & $5(35.7 \%)$ & 1.0 \\
No & $9(64.3 \%)$ & $9(64.3 \%)$ & \\
Contraceptive pills & $1 / 10$ & $2 / 9$ & 1.0 \\
Educational level & & & \\
Secondary & $4(28.6 \%)$ & $4(28.6 \%)$ & 0.875 \\
University & $8(57.1 \%)$ & $7(50 \%)$ & \\
Higher level & $2(14.3 \%)$ & $3(21.4 \%)$ & \\
\hline
\end{tabular}

${ }^{\text {a }}$ Values are mean $\pm \mathrm{SD}$

\section{Injection techniques}

Both fixed-site and follow-the-pain approaches were adopted in the present study. Doses of BTX-A ranged from 30 to $80 \mathrm{IU}$ with a mean of $(50.14 \pm 13.51)$. In all patients, frontalis and temporalis muscles were either unilaterally or bilaterally injected, the less frequently injected muscles were sternocleidomastoideus.

Outcome measures

\section{Primary efficacy variables}

There was a significant reduction in both number of headache days per month (frequency), and headache severity measured by VAS, and significant improvement of QoL assessed by HDI in BTX-A group at 30 and 90 days assessment when compared to the placebo group. (Table 4). Upon assessment of changes from baseline at 90 days, there was $37.75 \%$ reduction of headache days (in BTX-A) group versus $17.13 \%$ (in placebo) (Fig. 1), $43.68 \%$ decrease in headache severity as assessed by VAS (in BTX-A group) versus $17.98 \%$ (in placebo), and $40.57 \%$
Table 4 Primary efficacy variables at baseline, 30 and 90 days of study population

\begin{tabular}{|c|c|c|c|c|c|}
\hline & \multicolumn{2}{|l|}{ BTX } & \multicolumn{2}{|c|}{ Placebo } & \multirow[t]{2}{*}{$P$ value } \\
\hline & Mean & SD & Mean & SD & \\
\hline \multicolumn{6}{|c|}{ Headache (days/months) } \\
\hline Baseline & 19.93 & 3.75 & 19.21 & 3.17 & 0.591 \\
\hline 30 days & 15.00 & 2.25 & 17.50 & 2.03 & 0.005 \\
\hline 90 days & 12.07 & 1.94 & 15.92 & 2.16 & 0.000 \\
\hline \multicolumn{6}{|c|}{ Headache severity (VAS) } \\
\hline Baseline & 6.21 & 1.05 & 6.36 & 1.08 & 0.726 \\
\hline 30 days & 4.79 & 1.05 & 5.86 & 0.86 & 0.007 \\
\hline 90 days & 3.50 & 1.22 & 5.21 & 1.19 & 0.001 \\
\hline \multicolumn{6}{|l|}{ HDI } \\
\hline Baseline & 64.43 & 8.74 & 60.57 & 10.27 & 0.294 \\
\hline 30 days & 44.29 & 14.84 & 56.14 & 11.70 & 0.027 \\
\hline 90 days & 38.29 & 19.84 & 56.57 & 12.31 & 0.007 \\
\hline
\end{tabular}

decrease in HDI (in BTX-A group) versus $6.60 \%$ (in placebo group).

\section{Secondary efficacy variables}

There was a significant decrease in the duration of headache per day (hours), and number of days with acute headache medications per month in BTX-A group at 30and 90-day assessment when compared to the placebo group. (Table 5). Upon assessment of changes from baseline at 90 days, there was $26 \%$ decrease of duration of headache per day (hours) (in BTX-A) group versus $14.56 \%$ (in placebo) (Fig. 2), and $42.28 \%$ decrease in number of days with acute headache medications per month (in BTXA group) versus $21.94 \%$ (in placebo).

Adverse events

Treatments were generally tolerated, no systemic reactions were noted and there were no serious injections-related adverse events. The reported side effects were hematoma at the site of injection, blepharoptosis, itching and pain at the
Table 3 Baseline clinical profile of the study population

\begin{tabular}{lrrr}
\hline & \multicolumn{1}{c}{ BTX-A } & \multicolumn{1}{c}{ Placebo } & $P$ value \\
\hline Headache duration (years) & $4.86 \pm 2.93$ & $4.71 \pm 2.27$ & 0.886 \\
Duration of prophylactic treatment (months) & $15.93 \pm 7.78$ & $16.36 \pm 7.48$ & 0.883 \\
Headache days/months & $19.92 \pm 3.75$ & $19.21 \pm 3.17$ & 0.591 \\
Duration of headache per day (h) & $8.50 \pm 1.09$ & $8.86 \pm 1.03$ & 0.381 \\
Number of days with acute headache medications/months & $11.14 \pm 2.59$ & $10.71 \pm 2.33$ & 0.650 \\
Headache severity (VAS) & $6.21 \pm 1.05$ & $6.36 \pm 1.08$ & 0.726 \\
HDI (baseline) & $64.43 \pm 8.74$ & $60.57 \pm 10.27$ & 0.294 \\
\hline
\end{tabular}




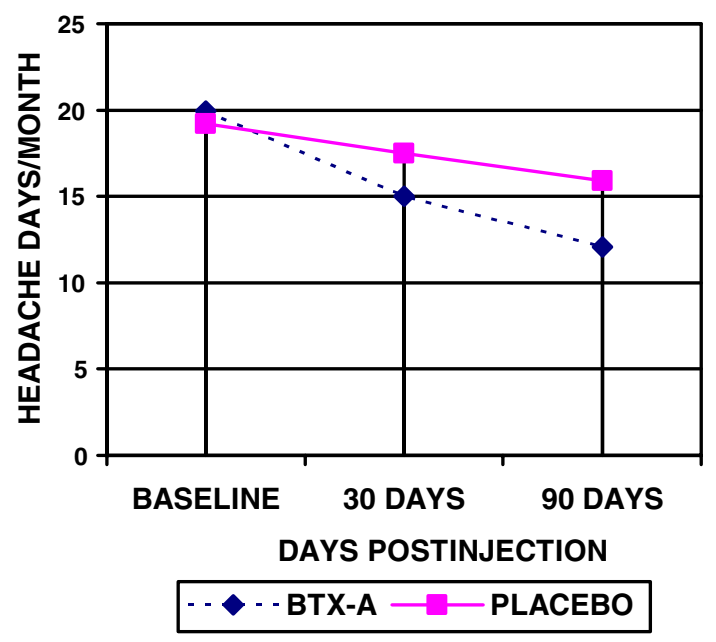

Fig. 1 Changes of headache days/months from baseline at 30 and 90 days

Table 5 Secondary efficacy variables at baseline, 30 and 90 days of study population

\begin{tabular}{|c|c|c|c|c|c|}
\hline & \multicolumn{2}{|l|}{ BTX } & \multicolumn{2}{|c|}{ Placebo } & \multirow[t]{2}{*}{$P$ value } \\
\hline & Mean & SD & Mean & SD & \\
\hline \multicolumn{6}{|c|}{ Duration of headache per day (h) } \\
\hline Baseline & 8.50 & 1.09 & 8.86 & 1.03 & 0.381 \\
\hline 30 days & 6.93 & 0.83 & 8.07 & 0.92 & 0.002 \\
\hline 90 days & 6.29 & 0.91 & 7.57 & 1.34 & 0.006 \\
\hline \multicolumn{6}{|c|}{ Number of days with acute headache medications/months } \\
\hline Baseline & 11.14 & 2.59 & 10.71 & 2.33 & 0.650 \\
\hline 30 days & 7.43 & 1.09 & 9.64 & 2.02 & 0.001 \\
\hline 90 days & 6.43 & 1.16 & 8.36 & 1.65 & 0.001 \\
\hline
\end{tabular}

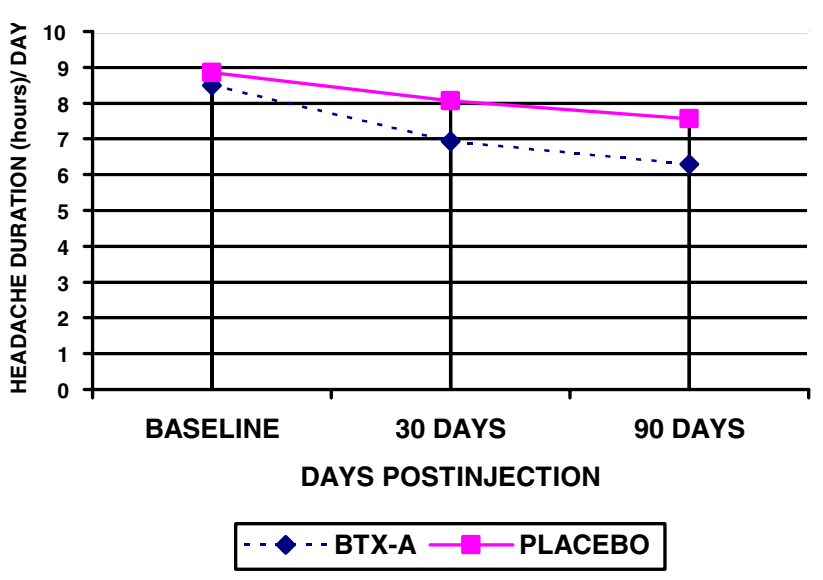

Fig. 2 Changes of headache duration/day from baseline at 30 and 90 days

site of injection (Table 6). All these side effects were transitory and did not interfere with the patient activity, and did not need further management.
Table 6 Treatment-related adverse events

\begin{tabular}{lll}
\hline & BTX-A & Placebo \\
\hline Hematoma at site of injection & 1 & 1 \\
Blepharoptosis & 1 & 0 \\
Itching at site of injection & 0 & 1 \\
Pain at site of injection & 1 & 1 \\
\hline
\end{tabular}

\section{Discussion}

Based on the results of this clinical trial in 28 Egyptian patients with CTTH, we can conclude that botulinum toxin injection in a combination of both fixed-site and followthe-pain approaches is an effective and relatively safe treatment option in patients for whom standard prophylactic therapy has failed.

Several recent studies $[9,12,24-26]$ have demonstrated the effectiveness of botulinum toxin in treating several disorders related to muscle spasticity and pain, and have emphasized the potential role for this agent in management of different types of chronic primary headaches including migraine and CTTH.

Though there are large numbers of clinical studies for the evaluation of botulinum toxin in the treatment of TTH, yet, the results are contradictory. Many case reports and open-label studies have shown positive results, with significant improvement in different efficacy end points such as such as number of headache-free days, headache severity and duration, quality of life and tenderness to palpation [12, 27-29]. However, in different randomized, double-blind, placebo-controlled studies that were conducted in patients with TTH, there were conflicting and oftentimes equivocal results for the effectiveness of botulinum toxin [30]. While a significant improvement of headache was detected by Relja and Telarovic [31], Porta [6], and Smuts et al. [32], who concluded that BTX-A is an effective prophylactic treatment for TTH in patients for whom standard therapy has failed. Padberg et al. [33] found no significant differences between placebo and up to 100-IU botulinum toxin that were injected in muscles with increased tenderness in patients with CTTH in week 4, 8 and 12 post-injection. Same unsatisfactory responses were found by Rollnik et al. [15] and Schmitt et al. [34] who concluded that increased muscle tenderness might not be an important pathophysiology of CTTH. This disparity in the results of randomized trials could be attributed to several methodological factors that include variable injected doses of BTX-A in different studies, injection sites, which were not always standardized, and the used injection techniques that vary across studies and have not been consistently reported $[1,7]$. 
As regards injection techniques, some researchers used individual injection sites in the most tender muscles or trigger points (follow-the-pain approach), while in other studies there was a standardized injection design (fixed-site approach) [7]. However, Ewans and Blumenfeld [35] reported that follow-the-pain approach is more commonly used in chronic tension-type or chronic daily headache patients, while they recommended fixed-site approach for patients with migraine, because follow-the-pain technique may produce a suboptimal cosmetic outcome, and the headaches may shift to the previously unaffected side. In our study, we used a modified treatment paradigm using a combination of both methods, which was previously reported by Mathew [21]; the injected points were identified from the potential tender points in the frontal and temporal regions, and the majority of patients were injected in two nuchal points, close to the occipital origin of the trapezius muscle. The injected muscles included frontalis, temporalis, sternocleidomastoideus, trapezius, splenius capitis, and semispinalis [22]. The adopted modified technique in our study allowed us to select the most tender points from the commonly affected ones in those with CTTH without apparent detection of suboptimal cosmetic outcome.

It seems clear that there is no standardized points for injections in headache patients, and the selected protocol for injections and injection application techniques sometimes are regarded as important causes of lack of efficacy of BT-A, and a larger distribution of injection points seems compatible with better results [36].

In the current study, BTX-A is used in the range of 30$80 \mathrm{IU}$ with a mean of $(50.14 \pm 13.51 \mathrm{IU})$, and this goes in accordance with Rozen and Sharma [1] who reported that the range of doses of BTX-A used in positive studies ranged from 15 to $100 \mathrm{IU}$ (Botox) or 160 to $200 \mathrm{IU}$ of Dysport. Moreover, we injected BTX-A in small doses at multiple sites to reduce the occurrence of side effects as recommended by Ates [7]. To achieve this, a dilution of $4 \mathrm{~mL}$ of normal saline to 100 units of BTX-A was used. The injections were administered intramuscularly to limit discomfort and side effects that could occur by soft tissue diffusion as intradermal injections tend to be more uncomfortable [35]. In the placebo group we used the vial $(2 \mathrm{~mL} 0.9 \mathrm{NaCl})$, and patients received equal volumes of saline that patients received.

Other factor to consider is the diagnostic criteria of the included patients, some studies of the prophylactic effect of BTX-A on headache had a broad headache diagnosis, and others included heterogeneous group of headache disorders [27]. Klapper et al. [37] in their double-blind, placebocontrolled trial that enrolled 56 patients, and Robbins [38] in an open-label study that included 79 patients used "chronic daily headache" as a diagnosis of included patients. In our study, we included patients with CTTH, and the diagnosis was established strictly following the criteria of the HIS, and patients were eligible in this study when they had history of failed treatment in the last 3 months with at least one prophylactic drug, and to have the ability to distinguish between the different headache types.

In the current study, we specified the outcome measures as either primary or secondary efficacy variables; all efficacy measures showed significant improvement in the BTX-A group when assessed at 30 and 90 days following injection day. The primary outcome measures were headache frequency per month assessed by headache diary of the patient, and headache severity assessed by the standard visual analogue scale, which is a subjective measure of headache that represents the patient's perception of headache status. We also included quality of life as a primary efficacy point that was assessed by the 25 -item beta version HDI ( $\beta$-HDI), a test with strong internal consistency/reliability that used to quantify the impact of headache and its treatment, on daily living, the items in the $\beta$-HDI were subgrouped into either functional or emotional subscales [20]. Our secondary efficacy points were the duration of headache per day (hours), and number of days of symptomatic therapy per month. In our study, we precisely defined both primary and secondary efficacy points to eliminate a major part of methodological inconsistency that could lead to inconclusive results, and this was also recommended by Rozen and Sharma [1] who proposed that reduction of headache frequency should be adopted as a primary endpoint.

Various retrospective or well-designed controlled studies considered number of headache-free days (headache frequency) as a primary outcome measure [22, 26, 39-41], and some studies added the headache intensity (severity) [22]. The secondary efficacy measures in different trials were global impressions, the use of abortive headache medications and the proportion of patients with a decrease from baseline of $50 \%$ or more [26, 39, 41]. The determination of precise primary and secondary efficacy end points is one of major prerequisites to avoid contradiction in studies results. However, a point of consideration was the higher placebo response than what was expected; this effect was previously reported by Silberstein et al. [40].

The precise mechanism by which headache intensity and severity are reduced is not known, based on available data, BTX-A treatment might reduce the local release of nociceptive neuropeptides [16, 42] and have either direct or indirect effects on central sensitization [43]. In all patients treated in this study, the injection sites included facial and scalp regions in the distribution of the first branch of trigeminal nerve or cervical dermatome distribution which is believed to be important in the proposed action of BTX-A 
which is related to the neuromodulatory effect of afferent inputs to the trigeminal nucleus from the scalp, skin and muscle within these regions [42]. It is hypothesized that BTX-A has its effect not by direct muscle relaxation, but by effects on afferent inputs into the trigeminal nucleus $[16,42]$.

The follow-up period in the current study was 90 days during which patients showed a gradual and constant trend of improvement in their headache frequency and intensity; however, the duration of the effect of BTX-A for CTTH was not studied in the current trial beyond this period, because our study focused on one-session injection with 90 days follow-up period. A single injection might be enough to break the cycle of chronic headaches, and there would be no need for repeated injections $[42,44]$. The potential risk of antibody development with repeated injections and the cost of BTX-A were another major issue, because patients had to pay for it themselves. However, the cumulative effect of subsequent injections of botulinum toxin in tension headache was discussed by Ondo et al. [39] and Relja [45] who found a lasting positive effects in long-term botulinum toxin injections, as repeated injections had a step-like therapeutic effect and the consecutive therapeutic effect of each injection built on the effect previously achieved.

Results of our study have also shown that BTX-A could be safe and well-tolerated alternative therapy for CTTH. In the current patient series, there were no serious adverse events in the BTX-A group, and all detectable side effects were transitory, mild to moderate in severity. These included hematoma at the site of injection, blepharoptosis and local pain at the site of injection. These results were previously recognized in various reports that confirmed the safety and tolerability of BTX-A in headache patients [22, 26-28, 36, 39-42, 46].

In conclusion, BTX-A may be an effective and promising prophylactic treatment for CTTH in Egyptian patients, making it an alternative to patients in whom the standard therapeutic options have failed or can not be tolerated. However, several questions such as injection techniques, and doses and injection sites remain unsolved.

\section{Conflict of interest None.}

\section{References}

1. Rozen D, Sharma J (2006) Treatment of tension-type headache with botox: a review of the literature. Mt Sinai J Med 73(1):493498

2. Araki N (2005) Tension-type headache. Nippon Rinsho 63(10):1742-1746 (Abstract)

3. International Headache Society Classification Subcommittee (2004) International classification of headache disorders, 2nd edn. Cephalalgia 24(suppl 1):1-160
4. Mathew NT (2006) Tension-type headache. Curr Neurol Neurosci Rep 6(2):100-105

5. Ashina S, Bendtsen L, Ashina M (2005) Pathophysiology of tension-type headache. Curr Pain Headache Rep 9(6):415-422

6. Porta M (2000) A comparative trial of botulinum toxin A and methylprednisolone for the treatment of tension-type headache. Curr Rev Pain 4:31-35

7. Ates Y (2006) Botulinum toxin for the treatment of headaches: a review of current practices and evidence based-data. Agri 18(3):5-11

8. Binder WJ, Brin MF, Blitzer A, Schoenrock LD, Pogoda JM (2000) Botulinum toxin type A (Botox) for treatment of migraine headaches: an open-label study. Otolaryngol Head Neck Surg 123:669-676

9. Straube A, Empl M, Ceballos-Baumann A, Tolle T, Stefenelli U, Pfaffenrath V (2008) Pericranial injection of botulinum toxin type A (Dysport) for tension-type headache-a multicentre, doubleblind, randomized, placebo-controlled study. Eur J Neurol 15(3):205-213

10. Troost BT (2004) Botulinum toxin A (Botox) in the treatment of migraine and other headaches. Expert Rev Neurother 4(1):27-31

11. Gobel H, Heinze A, Heinze-Kuhn K, Jost WH (2001) Evidencebased medicine: botulinum toxin A in migraine and tension-type headache. J Neurol 248(Suppl 1):34-38

12. Schulte-Mattler WJ, Martinez-Castrillo JC (2006) Botulinum toxin therapy of migraine and tension-type headache: comparing different botulinum toxin preparations. Eur J Neurol 13(Suppl 1):51-54

13. Evers S, Rahmann A, Vollmer-Haase J, Husstedt IW (2002) Treatment of headache with botulinum toxin A-a review according to evidence based criteria. Cephalalgia 22:699-710

14. Wheeler AH (1998) Botulinum toxin A, adjunctive therapy for refractory headaches associated with pericranial muscle tension. Headache 38:468-471

15. Rollnik JD, Karst M, Fink M, Dengler R (2001) Botulinum toxin Type A and EMG: a key to the understanding of chronic tensiontype headaches? Headache 41(10):985-989

16. Durham PL, Cady R, Cady R (2004) Regulation of calcitonin gene-related peptide secretion from trigeminal nerve cells by botulinum toxin type A: implications for migraine therapy. Headache 44:35-43

17. Blumenfeld AJ (2004) Botulinum toxin type A: a neuromodulatory mechanism of action in migraine (commentary). Headache 44:42-43

18. Dressler D, Saberi FA, Barbosa ER (2005) Botulinum toxin: mechanisms of action. Arq Neuropsiquiatr 63:180-185

19. Scott J, Huskisson EC (1976) Graphic representation of pain. Pain 2:175-184

20. Jacobson GP, Ramadan NM, Aggarwal SK, Newman CW (1994) The Henry Ford Hospital Headache Disability Inventory (HDI). Neurology 44:837-842

21. Mathew N, Kallasam K, Meadors L (2002) Disease modification in chronic migraine with botulinum toxin type-A-long term experience. Headache 42:389-463

22. Blumenfeld A (2003) Botulinum toxin type A as an effective prophylactic treatment in primary headache disorders. Headache 43(8):853-860

23. Evers R, Vollmer-Haase J, Schwaag S, Rahmann A, Husstedt IW, Frese A (2004) Botulinum toxin A in the prophylactic treatment of migraine-a randomized, double-blind, placebo-controlled study. Cephalalgia 24:838-843

24. Ashkenazi A, Silberstein S (2008) Botulinum toxin type A for the treatment of headache: why we say yes. Arch Neurol 65(1):146-149

25. Martin-Araguz A, Garrido-Carrion A, Fernandez-Armayor V (2007) Prophylaxis of chronic daily headache with a simplified 
regimen of subcutaneous administration of botulinum toxin type A. Rev Neurol 45(7):385-388. (Abstract). Spanish

26. Mathew NT, Frishberg BM, Gawel M, Dimitrova R, Gibson J, Turkel C, BOTOX CDH study group (2005) Botulinum toxin type A (BOTOX) for the prophylactic treatment of chronic daily headache: a randomized, double-blind, placebo-controlled trial. Headache 45(4):293-307

27. Suzuki K, Iizuka T, Sakai F (2007) Botulinum toxin type A for migraine prophylaxis in the Japanese population: an open-label prospective trial. Intern Med 46(13):959-963

28. Kemal Erdemoglu A, Varlibas A (2007) The long-term efficacy and safety of botulinum toxin in refractory chronic tension-type headache. J Headache Pain 8(5):294-300

29. Eross EJ, Gladstone JP, Lewis S, Rogers R, Dodick DW (2005) Duration of migraine is a predictor for response to botulinum toxin type A. Headache 45(4):308-314

30. Schulte-Mattler WJ, Leinisch E (2008) Evidence based medicine on the use of botulinum toxin for headache disorders. J Neural Transm 115(4):647-651

31. Relja M, Telarovic S (2004) Botulinum toxin in tension-type headache. J Neurol 251(Suppl 1):12-14

32. Smuts JA, Baker MK, Smuts HM, Rossouw E, DeGryse RE, Barnard PWA (1999) Prophylactic treatment of chronic tensiontype headache using botulinum toxin type A. Eur J Neurol 6(Suppl 4):99-102

33. Padberg M, de Bruijn SF, de Haan RJ, Tavy DL (2004) Treatment of chronic tension-type headache with botulinum toxin: a double-blind, placebo-controlled clinical trial. Cephalalgia 24(8):675-680

34. Schmitt WJ, Slowey E, Fravi N, Weber S, Burgunder JM (2001) Effect of botulinum toxin A injections in the treatment of chronic tension-type headache: a double-blind, placebo-controlled trial. Headache 41(7):658-664

35. Ewans RW, Blumenfeld A (2003) Botulinum toxin injections for headache. Headache 43:682-685
36. Menezes C, Rodrigues B, Magalhaes E, Melo A (2007) Botulinum toxin type A in refractory chronic migraine. An open-label trial. Arq Neuropsiquiatr 65(3-A):596-598

37. Klapper JA, Mathew NT, Klapper A, Kailasam J (2000) Botulinum toxin type A (Btx-A) for the prophylaxis of chronic daily headache. Cephalalgia 20:292-293

38. Robbins L (2001) Botulinum toxin A for refractory chronic daily headache. Neurology 56(Suppl 3):A349

39. Ondo WG, Vuong KD, Derman HS (2004) Botulinum toxin A for chronic daily headache: a randomized, placebo-controlled, parallel design study. Cephalalgia 24(1):60-65

40. Silberstein SD, Stark SR, Lucas SM, Christie SN, Degryse RE, Turkel CC, BoNTA-039 Study Group (2005) Botulinum toxin type A for the prophylactic treatment of chronic daily headache: a randomized, double-blind, placebo-controlled trial. Mayo Clin Proc 80(9): 1126-1137

41. Aurora SK, Gawel M, Brandes JL, Pokta S, Vandenburgh AM, BOTOX North American Episodic Migraine Study Group (2007) Botulinum toxin type a prophylactic treatment of episodic migraine: a randomized, double-blind, placebo-controlled exploratory study. Headache 47(4):486-499

42. Liu Y-C, Fuh J-L, Chen R-C, Lin K-P, Wang S-J (2007) Botulinum toxin Type $\mathrm{A}$ in the prophylactic treatment of transformed migraine in Taiwanese patients: a review of 30 consecutive cases. J Chin Med Assoc 70(12):535-540

43. Aoki KR (2003) Evidence for antinociceptive activity of botulinum toxin type A in pain management. Headache 43(Suppl):9-15

44. Mauskop A (2002) The use of botulinum toxin in the treatment of headaches. Curr Pain Headache Rep 6:320-323

45. Relja MA (2000) Treatment of tension-type headache with botulinum toxin: 1-year follow-up. Cephalalgia 20:336

46. Mathew NT, Kailasam J, Meadors L (2008) Predictors of response to botulinum toxin type A (BoNTA) in chronic daily headache. Headache 48(2):194-200 